\title{
SOME PROPERTIES OF THE ANKENY-ONISHI FUNCTION
}

Harold G. Diamond \& Heini Halberstam

In honor of the 60th birthday of our friend, Jean-Marc Deshouillers

Abstract: We survey properties of the Ankeny-Onishi sieve function and establish inequalities for $j_{\kappa}(\kappa)$ and for $1-j_{\kappa}(u)$ for $u \rightarrow \infty$.

Keywords: sieves, recurrence, adjoint function.

\section{Introduction}

The function $\sigma_{\kappa}(u)$ was first introduced by Ankeny and Onishi in their pioneering extension [1] of the Selberg sieve method, albeit in a different notational guise. It is given by

$$
\sigma_{\kappa}(u):=j_{\kappa}(u / 2), \quad \kappa \geqslant 1
$$

where

$$
j(u)=j_{\kappa}(u)= \begin{cases}0, & u \leqslant 0, \\ e^{-\gamma \kappa} u^{\kappa} / \Gamma(\kappa+1), & 0<u \leqslant 1,\end{cases}
$$

and $j$ is continued forward as the continuous solution of

$$
u j^{\prime}(u)=\kappa j(u)-\kappa j(u-1)=\kappa \int_{u-1}^{u} j^{\prime}(t) d t, \quad u>1,
$$

by means of the restatement

$$
\left(u^{-\kappa} j(u)\right)^{\prime}=-\kappa u^{-\kappa-1} j(u-1), \quad u>1,
$$

of (1.3); in fact (1.3) holds for all $u \geqslant 0$. It is a differential delay equation of a kind common in the study of sieves.

In this note we review basic information about $j / \sigma$ and develop several interesting properties of these functions. In particular, we present simpler proofs that (i) $j_{\kappa}(\kappa)>1 / 2$ for all $\kappa \geqslant 1$, and that (ii) for each fixed $c>1, j_{\kappa}(c \kappa)$ tends 
to 1 from below as $\kappa \rightarrow \infty$ (both these results were first proved by Grupp and Richert in [2]); also, we show in explicit fashion that $j_{\kappa}(u) \rightarrow 1$ and $j_{\kappa}^{\prime}(u) \rightarrow 0$ as $u \rightarrow \infty$, each at a rate that is faster than exponential.

We begin studying $j$ with some observations about the continuity of its derivatives. If $u>0$ and $\kappa \geqslant 1$, then $j^{\prime}(u)$ is continuous for $u>0$ by (1.3) and the continuity of $j$; more generally, by differentiating (1.3) we see that $j_{\kappa}^{(n)}(u)$ is continuous for $u \geqslant 0$ for all positive integers $n<\kappa$. If $\kappa$ is a positive integer, then $j_{\kappa}^{(\kappa)}(u)$ has a jump discontinuity at $u=0$, and $j_{\kappa}^{(\kappa+n)}(u)$ has jump discontinuities at $u=1, \ldots, n$. If $\kappa>1$ is not an integer, then $j^{([\kappa]+n)}(u)$ has infinite jump discontinuities from the right at $u=0,1, \ldots, n-1$ for each positive integer $n$. In each of the preceding cases, the function is continuous at all other values of $u>0$.

We show next for each $\kappa \geqslant 1$ that $j_{\kappa}(u)$ is a positive, strictly increasing function of $u>0$. By (1.2), $j^{\prime}(u)>0$ when $0<u \leqslant 1$, and by (1.3) it remains positive for some distance to the right side of 1 . Suppose there were a point $u_{0}>1$ with $j^{\prime}\left(u_{0}\right)=0$. By the continuity of $j^{\prime}$, we may assume that $u_{0}$ is the first such point, i.e. that $j^{\prime}\left(u_{0}\right)=0$ and $j^{\prime}(t)>0$ for $0<t<u_{0}$. Upon evaluating the integral form of (1.3) at $u=u_{0}$ we obtain a contradiction, since the left side is 0 and the right side is $\kappa$ times the integral of a positive function. Hence

$$
j^{\prime}(u)>0, \quad u>0
$$

and we deduce immediately that

$$
j(u)>0, \quad u>0 .
$$

The higher derivatives of $j(u)$ also satisfy differential delay equations. Upon differentiating (1.3), and then once again, we obtain

$$
u j^{\prime \prime}(u)=(\kappa-1) j^{\prime}(u)-\kappa j^{\prime}(u-1)
$$

and

$$
u j^{\prime \prime \prime}(u)=(\kappa-2) j^{\prime \prime}(u)-\kappa j^{\prime \prime}(u-1) .
$$

In (1.3) itself, if we integrate by parts on the right (which is valid, since $j^{\prime}$ is absolutely continuous), we obtain

$$
u j^{\prime}(u)=\left.\kappa(t-\kappa+1) j^{\prime}(t)\right|_{u-1} ^{u}-\kappa \int_{u-1}^{u}(t-\kappa+1) j^{\prime \prime}(t) d t
$$

or

$$
(u-\kappa)\left\{(\kappa-1) j^{\prime}(u)-\kappa j^{\prime}(u-1)\right\}=\kappa \int_{u-1}^{u}(t-\kappa+1) j^{\prime \prime}(t) d t ;
$$

hence by (1.6) (for all $\kappa \geqslant 1$ and $u>0$ ),

$$
u(u-\kappa) j^{\prime \prime}(u)=\kappa \int_{u-1}^{u}(t-\kappa+1) j^{\prime \prime}(t) d t .
$$

We use the last equation to show that $j_{\kappa}$ has a unique inflection point $u_{\kappa}$ (for $\kappa>1)$ and that it lies in the interval $(\kappa-1, \kappa]$. A finer analysis (see [2]) would show that $\kappa-1 / 2<u_{\kappa}<\kappa$ for all $\kappa>1$. 
Lemma 1. Suppose $\kappa>1$. There exists a unique number, call it $u_{\kappa}$, between $\kappa-1$ and $\kappa$, such that $j^{\prime \prime}(u)>0$ for $0<u<u_{\kappa}$ and $j^{\prime \prime}(u)<0$ for all $u>u_{\kappa}$. For $\kappa=1$, we have $j^{\prime \prime}(u)=0$ for all $u<u_{1}=\kappa=1$ and $j^{\prime \prime}(u)<0$ for all $u>1$.

Proof. For $\kappa=1$, we have by (1.6) that $u j^{\prime \prime}(u)=-j^{\prime}(u-1)$, an expression that is 0 for $u<1$ and is negative for $u>1$ by (1.5).

Now suppose $\kappa>1$. On taking $u=\kappa$ in (1.8) we find that

$$
\int_{\kappa-1}^{\kappa}(t-\kappa+1) j^{\prime \prime}(t) d t=0 .
$$

Since $t-\kappa+1>0$ on $(\kappa-1, \kappa)$ it follows that $j^{\prime \prime}(t)$ changes sign in this interval. By $(1.2) j^{\prime \prime}(u)>0$ on $(0,1]$ and it follows from (1.7) and the continuity of $j^{\prime}$ that $j^{\prime \prime}$ is continuous on $[0, \infty)$. Thus there exists some number $u_{\kappa}$, the smallest value of $u>1$ at which $j^{\prime \prime}(u)=0$. By (1.7) at $u=u_{\kappa}$

$$
u_{\kappa} j^{\prime \prime \prime}\left(u_{\kappa}\right)=-j^{\prime \prime}\left(u_{\kappa}-1\right)<0
$$

since $j^{\prime \prime}(u)>0$ for $0<u<u_{\kappa}$, whence $u_{\kappa}$ is a simple zero of $j^{\prime \prime}$.

Suppose if possible that $j^{\prime \prime}$ has other zero beyond $u_{\kappa}$, and let $v$ be the least of these. We claim that

$$
v<u_{\kappa}+1
$$

for if, on the contrary, $v \geqslant u_{\kappa}+1$ then $j^{\prime \prime}(v)=0$ and $j^{\prime \prime}(u)<0$ when $u_{\kappa}<u<v$. But then, by (1.6) at $u=v$,

$$
0=v j^{\prime \prime}(v)=(\kappa-1) j^{\prime}(v)-\kappa j^{\prime}(v-1)
$$

so that

$$
0<j^{\prime}(v)=\kappa\left\{j^{\prime}(v)-j^{\prime}(v-1)\right\}=\kappa j^{\prime \prime}(w)
$$

for some $w$ strictly between $v-1\left(\geqslant u_{\kappa}\right)$ and $v$, a contradiction.

Next suppose that $u_{\kappa}<v<u_{\kappa}+1$. We know that $j^{\prime \prime}(u)$ is non-decreasing at $u=v$, so that $j^{\prime \prime \prime}(v) \geqslant 0$; yet by $(1.7)$

$$
v j^{\prime \prime \prime}(v)=-\kappa j^{\prime \prime}(v-1)<0
$$

since $v-1<u_{\kappa}$, also an impossibility.

Hence $v$ does not exist, and $j^{\prime \prime}$ has just the one zero $u_{\kappa}$, which is simple and lies in $(\kappa-1, \kappa)$.

The most rapid rate of increase of $j$ occurs at $u_{\kappa}$. How fast is the function rising here? It was shown by Wheeler ([3], [4]) that $j_{\kappa}^{\prime}\left(u_{\kappa}\right) \sim 1 / \sqrt{\pi \kappa}$ as $\kappa \rightarrow \infty$. 


\section{The adjoint function}

We introduce next the so-called "adjoint" of $j$, a function $r(u)=r_{\kappa}(u)$ defined for $\kappa>0$ by

$$
(u r(u))^{\prime}=\kappa r(u+1)-\kappa r(u), \quad u>0,
$$

and normalized so that

$$
\lim _{u \rightarrow \infty} u r(u)=1 \text {. }
$$

A normalized solution of (2.1) is provided by

$$
r_{\kappa}(u)=\int_{0}^{\infty} \exp (-u t+\kappa \operatorname{Ein} t) d t
$$

where

$$
\operatorname{Ein} t:=\int_{0}^{t}\left(1-e^{-s}\right) \frac{d s}{s}=\sum_{n=1}^{\infty}(-1)^{n-1} \frac{t^{n}}{n ! n}, \quad t \in \mathbb{C}
$$

an entire function. With $\log t$ denoting the principal value of $\log t$,

$$
\operatorname{Ein} t=\log t+\gamma+\int_{t}^{\infty} \frac{e^{-s}}{s} d s, \quad|\arg t|<\pi
$$

To see that the integral (2.3) satisfies (2.1), first integrate it by parts, next multiply by $u$, and then differentiate with respect to $u$.

The behavior of $r(u)$ as $u \rightarrow \infty$ is no harder to derive: by (2.4) we have

$$
0 \leqslant \operatorname{Ein} t \leqslant t, \quad t \geqslant 0
$$

whence

$$
\int_{0}^{\infty} \exp (-u t) d t<r_{\kappa}(u)<\int_{0}^{\infty} \exp (-u t+\kappa t) d t
$$

and it follows at once that

$$
u^{-1}<r_{\kappa}(u) \quad(u>0) \text { and } r_{\kappa}(u)<(u-\kappa)^{-1} \quad(u>\kappa) .
$$

Together, the last two inequalities imply that the normalization (2.2) holds.

The integral representation $(2.3)$ of $r(u)$ shows that $(-1)^{\nu} r^{(\nu)}(u)>0$ for $\nu=0,1,2, \ldots$, and in particular, that $r^{\prime}(u)<0$ and $r^{\prime \prime}(u)>0$ for all $u>0$; also, that $(u r(u))^{\prime}<0$ by $(2.1)$ and $((u-\kappa) r(u))^{\prime}>0$. The last inequality holds since

$$
((u-\kappa) r(u))^{\prime}=\kappa\left\{r(u+1)-r(u)-r^{\prime}(u)\right\}=\kappa r^{\prime \prime}(u+\theta) / 2>0
$$

for some $\theta$ in $(0,1)$, by Taylor's expansion. It follows that

$$
\frac{u+1}{u}<\frac{r(u)}{r(u+1)}<\frac{u-\kappa+1}{u-\kappa},
$$

the latter for $u>\kappa$. 
The Iwaniec "inner product"

$$
\langle j, r\rangle(u):=u j(u) r(u)-\kappa \int_{u-1}^{u} j(t) r(t+1) d t, \quad u>0
$$

is constant, as one can verify by differentiating and using the defining equations of $r$ and $j$. To evaluate this constant let $u \rightarrow 0+$; by (2.3) and (2.5)

$$
\begin{aligned}
r(u)= & \int_{0}^{\infty} \exp \{-u t+\kappa \log t+\gamma \kappa+o(1)\} d t \\
& \sim e^{\gamma \kappa} \int_{0}^{\infty} \exp (-u t) t^{\kappa} d t, \quad u \rightarrow 0+, \\
= & e^{\gamma \kappa} \Gamma(\kappa+1) u^{-\kappa-1} .
\end{aligned}
$$

Hence, by (1.2), $u j(u) r(u) \rightarrow 1$ as $u \rightarrow 0+$ and so

$$
u j(u) r(u)-\kappa \int_{u-1}^{u} j(t) r(t+1) d t=1, \quad u>0 .
$$

In the same vein

$$
u r(u)-\kappa \int_{u-1}^{u} r(t+1) d t
$$

is constant by (2.1), and since $u r(u) \rightarrow 1$ as $u \rightarrow \infty$, we see that

$$
u r(u)-\kappa \int_{u-1}^{u} r(t+1) d t=1 .
$$

Lemma 2. Suppose $\kappa \geqslant 1$ and $u \geqslant \kappa$. Then each of the functions

$$
(j(u)-j(t)) r(t+1),(1-j(t)) r(t+1)
$$

is convex in $t$ on the interval $u-1 \leqslant t \leqslant u$.

Proof. The argument is the same for each function, so focus on the first and call it $J(t)$. Then, by $(2.3)$,

$$
\begin{aligned}
J^{\prime \prime}(t)= & -r(t+1) j^{\prime \prime}(t)+2\left(-j^{\prime}(t)\right) r^{\prime}(t+1)+(j(u)-j(t)) r^{\prime \prime}(t+1) \\
= & \int_{0}^{\infty}\left\{-j^{\prime \prime}(t)+2\left(-j^{\prime}(t)\right)(-u)+(j(u)-j(t)) u^{2}\right\} \\
& \times \exp (-(t+1) u+\kappa \operatorname{Ein} u) d u .
\end{aligned}
$$

By (1.6) the expression within the curly brackets is equal to

$$
\begin{gathered}
-\frac{1}{t}\left((\kappa-1) j^{\prime}(t)-\kappa j^{\prime}(t-1)\right)+2 u j^{\prime}(t)+(j(u)-j(t)) u^{2} \\
=\left(2 u-\frac{\kappa-1}{t}\right) j^{\prime}(t)+\frac{\kappa}{t} j^{\prime}(t-1)+(j(u)-j(t)) u^{2}
\end{gathered}
$$


The second and third terms here are positive, and the coefficient of $j^{\prime}(t)$ is at least

$$
2 u-\frac{\kappa-1}{u-1} \geqslant 2 u-1>0
$$

since $u \geqslant \kappa$. Hence, $J^{\prime \prime}>0$.

We next consider the limiting behavior of $j(u)$ as $u \rightarrow \infty$. When we multiply $(2.7)$ by $j(u)$ and subtract it from (2.6) we obtain

$$
1-j(u)=\kappa \int_{u-1}^{u}\{j(u)-j(t)\} r(t+1) d t
$$

if we simply subtract (2.6) from (2.7) this time we find

$$
\{1-j(u)\} u r(u)=\kappa \int_{u-1}^{u}\{1-j(t)\} r(t+1) d t .
$$

There is much to be learned from these two relations. First, the integral on the right of (2.8) is positive and therefore

$$
j(u)<1, \quad u>0
$$

as we reported earlier. Next, by $(2.9)$, since $r(\cdot)$ is positive and decreasing and $j(\cdot)>0$, we obtain at once

$$
\{1-j(u)\} \operatorname{ur}(u)<\kappa \int_{u-1}^{u} r(t+1) d t<\kappa r(u),
$$

so that

$$
0<1-j(u)<\kappa / u
$$

and therefore

$$
\lim _{u \rightarrow \infty} j_{\kappa}(u)=1 \text {. }
$$

We apply Lemma 2 to the right side of (2.9) and obtain

$$
\{1-j(u)\} u r(u)<\frac{\kappa}{2}(r(u+1)\{1-j(u)\}+r(u)\{1-j(u-1)\}), \quad u \geqslant \kappa ;
$$

this inequality can be rewritten in two ways, which lead to different lines of development, one an iteration and the other a differential inequality.

First, we have

$$
\begin{aligned}
1-j(u) & <\frac{\kappa r(u)}{2 u r(u)-\kappa r(u+1)}\{1-j(u-1)\} \\
& =\frac{\kappa}{2 u-\kappa r(u+1) / r(u)}(1-j(u-1))
\end{aligned}
$$


and since $r(u+1) / r(u)<u /(u+1)$ from above, we derive the recurrence

$$
1-j(u)<\frac{u+1}{u} \frac{\kappa / 2}{u+1-\kappa / 2}\{1-j(u-1)\}, \quad u \geqslant \kappa .
$$

This inequality plainly lends itself to iteration and leads, for any $v \geqslant \kappa-1$ and positive integer $n$, to

$$
\begin{aligned}
1- & j_{\kappa}(v+n) \\
& <\frac{v+1-\kappa / 2}{v+1} \frac{v+n+1}{v+n+1-\kappa / 2} \frac{(\kappa / 2)^{n} \Gamma(v+1-\kappa / 2)}{\Gamma(v+n+1-\kappa / 2)}\left(1-j_{\kappa}(v)\right) \\
& <\Gamma(v+1-\kappa / 2)\left\{\frac{(\kappa / 2)^{n}}{\Gamma(v+n+1-\kappa / 2)}\right\}\left(1-j_{\kappa}(v)\right) .
\end{aligned}
$$

If $u$ is a number near $\kappa+n$ for some positive integer $n$, then the factor in curly brackets shows that $j_{\kappa}(u)$ does indeed tend to 1 faster than exponentially as $u \rightarrow \infty$. In the next section we shall show that $1-j_{\kappa}(\kappa)<1 / 2$, which in combination with $(2.13)$ yields a quite sharp inequality for $1-j_{\kappa}(u)$.

To conclude this section, we return to $(2.11)$ and deduce from it a differential inequality. We begin by writing the relation in the form

$$
\{1-j(u)\} u r(u)<\frac{\kappa}{2}\{1-j(u)\}(r(u+1)+r(u))+\frac{\kappa}{2}(\{j(u)-j(u-1)\} r(u)
$$

and, after applying (1.3) and a little rearrangement, this becomes

$$
\begin{aligned}
1-j(u) & <\frac{\kappa}{2}(1-j(u))\left(\frac{r(u+1)}{u r(u)}+\frac{1}{u}\right)+\frac{1}{2} j^{\prime}(u) \\
& <\frac{\kappa}{2}(1-j(u))\left(\frac{1}{u+1}+\frac{1}{u}\right)+\frac{1}{2} j^{\prime}(u),
\end{aligned}
$$

or

$$
(1-j(u))^{\prime}+\left\{2-\kappa\left(\frac{1}{u}+\frac{1}{u+1}\right)\right\}(1-j(u))<0
$$

in other words, for $u \geqslant \kappa$,

$$
\left\{(1-j(u) \exp (2 u-\kappa \log u(u+1))\}^{\prime}<0 .\right.
$$

Upon integrating, we find for $u \geqslant \kappa$ that

$$
(1-j(u)) \exp (2 u-\kappa \log u(u+1)) \leqslant(1-j(\kappa)) \exp (2 \kappa-\kappa \log \kappa(\kappa+1)) .
$$

Here then we have come to a curious pass: starting from (2.11) and adding extra information - application of (1.3) - we have derived the inequality

$$
1-j_{\kappa}(u) \leqslant\left(1-j_{\kappa}(\kappa)\right)\left(\frac{u(u+1)}{\kappa(\kappa+1)}\right)^{\kappa} \exp (-2 u+2 \kappa), \quad u \geqslant \kappa,
$$


which is perhaps more pleasing to the eye, and not without interest, but yields only exponential decay of $1-j_{\kappa}(u)$ towards 0 as $u \rightarrow \infty$ ! We cannot understand why, apparently, the second approach is inferior to the first.

It should be said at this point that [1] derives a slightly weaker inequality than (2.14) valid for $u \geqslant \kappa+1$. This is implicit in their formula (2.9) on p. 40.

In the next section we shall simplify (2.13) and (2.14) by determining a lower bound for $j_{\kappa}(\kappa)$.

\section{A lower bound for $j_{\kappa}(\kappa)$}

We learn from (1.2) that $j_{1}(1)=e^{-\gamma}=0.56145 \ldots$ and from numerical computations that $j_{1.5}(1.5)=0.55179 \ldots$ and $j_{2}(2)=0.54454 \ldots$. In fact, it was proved in [2] that for any constant $c \geqslant 0, j_{\kappa}(\kappa+c)$ decreases in $\kappa \geqslant 1$ and tends to $1 / 2$ as $\kappa \rightarrow \infty$; also that $j_{\kappa}\left(c^{\prime} \kappa\right) \rightarrow 1$ as $\kappa \rightarrow \infty$ for any constant $c^{\prime}>1$. Also, it was shown by Wheeler ([3], [4]) that, for $\kappa \geqslant 1$,

$$
j_{\kappa}(\kappa)=1 / 2+1 /(9 \sqrt{\pi \kappa})+O\left(\kappa^{-3 / 2}\right) .
$$

Here we show by a Laplace inversion method that

Proposition 1. For $\kappa \geqslant 1$,

$$
j_{\kappa}(\kappa)>1 / 2 \text {. }
$$

Proof. Since $1-j_{\kappa}(u)$ vanishes rapidly at infinity, it has a Laplace transform whose integral converges for Res $\geqslant 0$. By a calculation analogous to that which identified $r(u)$ as a Laplace transform, we have

$$
\int_{0}^{\infty} e^{-s u}\left(1-j_{\kappa}(u)\right) d u=\frac{1}{s}(1-\exp (-\kappa \operatorname{Ein} s)), \quad \Re s \geqslant 0 .
$$

It follows by Fourier inversion (Laplace inversion on the imaginary axis) that, for $u>0$,

$$
1-j_{\kappa}(u)=\lim _{T \rightarrow \infty} \frac{1}{2 \pi} \int_{-T}^{T} e^{i u y}\{1-\exp (-\kappa \operatorname{Ein} i y)\} \frac{d y}{i y} .
$$

Since $j$ is real valued, we have at $u=\kappa$

$$
\begin{aligned}
1-j_{\kappa}(\kappa) & =\Re\left\{\frac{1}{2 \pi i} \int_{-\infty}^{\infty} e^{i \kappa y}(1-\exp \{-\kappa \operatorname{Ein}(i y)\}) \frac{d y}{y}\right\} \\
& =\frac{1}{\pi} \int_{0}^{\infty} \sin \kappa y \frac{d y}{y}-\Re\left\{\frac{1}{2 \pi i} \int_{-\infty}^{\infty} e^{-\kappa(\operatorname{Ein}(i y)-i y)} \frac{d y}{y}\right\} .
\end{aligned}
$$

The first expression on the right is well known to be equal to $1 / 2$. In the second expression,

$$
\begin{aligned}
\operatorname{Ein}(i y)-i y & =\int_{0}^{y} \frac{1-\cos t}{t} d t+i \int_{0}^{y} \frac{\sin t-t}{t} d t \\
& =C(y)+i S(y)
\end{aligned}
$$


say, where $C(y)$ is an even function of $y$ and $S(y)$ an odd function. Hence

$$
j_{\kappa}(\kappa)-\frac{1}{2}=\frac{1}{\pi} \int_{0}^{\infty} e^{-\kappa C(y)} \sin (-\kappa S(y)) \frac{d y}{y} .
$$

We complete the proof by showing that the integral on the right is positive.

Since

$$
\sin (-\kappa S(y))=\left(\kappa \frac{\sin y-y}{y}\right)^{-1} \frac{d}{d y} \cos (-\kappa S(y)),
$$

the integral equals, after integrating by parts,

$$
\left.\frac{1}{\kappa}\left\{\frac{e^{-\kappa C(y)}}{y-\sin y}(1-\cos \{\kappa S(y)\})\right\}\right|_{0} ^{\infty}-\frac{1}{\kappa} \int_{0}^{\infty}(1-\cos \{\kappa S(y)\}) \frac{d}{d y}\left(\frac{e^{-\kappa C(y)}}{y-\sin y}\right) d y .
$$

The integrated term vanishes at infinity since $C(y) \sim \log y$ as $y \rightarrow \infty$, and it vanishes also at 0 since

$$
1-\cos (\kappa S(y)) \sim \frac{1}{2 !}(\kappa S(y))^{2} \sim \frac{\kappa^{2}}{648} y^{6} \quad \text { as } y \rightarrow 0
$$

whereas

$$
y-\sin y \sim \frac{1}{6} y^{3} \quad \text { as } y \rightarrow 0 .
$$

As for the integral, we observe that each of $e^{-\kappa C(y)}$ and $(y-\sin y)^{-1}$ is positive and decreasing as $y$ increases, so that

$$
-\frac{d}{d y}\left(\frac{e^{-\kappa C(y)}}{y-\sin y}\right)>0 .
$$

Since $1-\cos (\kappa S(y))>0$, this completes the proof that the integral on the right side of (3.1) is positive.

The estimate of the Proposition appears to be quite sharp: it is likely, on the basis of the two asymptotic estimates of Wheeler that we have cited, that $j_{\kappa}(\kappa-1)<1 / 2$. However, we have not investigated this question.

The Proposition allows us to derive from (2.13) and (2.14)

Theorem 1. For $u \geqslant \kappa$

$$
j_{\kappa}(u) \geqslant 1-\frac{1}{2}\left(\frac{u(u+1)}{\kappa(\kappa+1)}\right)^{\kappa} \exp (2 \kappa-2 u),
$$

and for any positive integer $n$,

$$
\begin{aligned}
j_{\kappa}(n+\kappa) & >1-\frac{1}{2}\left(1-\frac{\kappa}{2 \kappa+2}\right) \Gamma\left(\frac{\kappa}{2}\right)\left(1+\frac{\kappa}{2 n+2+\kappa}\right) \frac{(\kappa / 2)^{n+1}}{\Gamma(n+1+\kappa / 2)} \\
& >1-\frac{\Gamma(\kappa / 2)(\kappa / 2)^{n+1}}{2 \Gamma(n+1+\kappa / 2)} .
\end{aligned}
$$


Corollary 1. Let $c>1$ be a constant. Then $j_{\kappa}(c \kappa) \rightarrow 1$ from below as $\kappa \rightarrow \infty$.

Proof. Let $c=1+\delta, \delta>0$. By $(3.2)$

$$
1>j_{\kappa}(c \kappa)>1-\frac{1}{2}(1+\delta)^{2 \kappa} \exp (-2 \kappa \delta)=1-\frac{1}{2}\left(\frac{1+\delta}{e^{\delta}}\right)^{2 \kappa} \rightarrow 1
$$

as $\kappa \rightarrow \infty$

The theorem is most effective when $u$ is large. As an illustration of its use, we have $\sigma_{\kappa}(3.5 \kappa)=j_{\kappa}(1.75 \kappa)>0.99995$ for $\kappa \geqslant 25$. In an earlier paper, we had been able to show only that $\sigma_{\kappa}(3.5 \kappa)>0.99994$ when $\kappa \geqslant 200$.

The following examples illustrate the accuracy - and the limitations - of formulas (3.2) and (3.3) for $\kappa$ and $u$ of modest size. For $\kappa=2$ and $u=6$ we have

$$
\begin{aligned}
1-j_{2}(6) & <0.00821 \ldots(\operatorname{using}(3.2)) \\
& <0.00324 \ldots(\operatorname{using}(3.3)-\text { first form }) \\
& =0.000908 \ldots(\text { calculation })
\end{aligned}
$$

We had remarked earlier that the differential inequality for $j$ gave poorer estimates than did the recurrence. We note in conclusion that estimates of $j^{\prime}(u)$ as $u \rightarrow \infty$ of the quality of (3.3) are easy to achieve. By (1.3)

$$
\begin{aligned}
u j^{\prime}(u) & =\kappa(1-j(u-1))-\kappa(1-j(u)) \\
& <\kappa(1-j(u-1)), \quad u>1 .
\end{aligned}
$$

In light of (2.12), little has been lost by omitting the term involving $1-j(u)$ when $u$ is large. Thus when $n \geqslant 1$, we have

$$
j_{\kappa}^{\prime}(n+1+\kappa)<\frac{\kappa}{n+1+\kappa}\left(1-j_{\kappa}(n+\kappa)\right),
$$

and we may apply (3.3) to estimate the last factor.

Added in proof. At the end of Section 2, we observed that the asymptotic estimate $(2.14)$ for $1-j(u)$ produced by using the differential equation was worse than that found by using the recursion (2.13). We have now obtained an estimate for $1-j(u)$ having the size predicted by the recursion. The method is based on establishing a monotonicity of $j^{\prime \prime} / j^{\prime}$. The details will be given in our forthcoming monograph on sieves.

\section{References}

[1] N.C. Ankeny and H. Onishi, The general sieve, Acta Arith. X (1964), 31-62.

[2] F. Grupp and H.-E. Richert, Notes on functions connected with sieve, Analysis 8 (1988), 1-23. 
[3] Ferrell S. Wheeler, On two differential-difference equations arising in analytic number theory, Ph.D. thesis, University of Illinois, Urbana, 1988.

[4] Ferrell S. Wheeler, On two differential-difference equations arising in number theory, Trans. A.M. S. 318 (1990), 491-521.

Address: Dept. of Math., Univ. of Illinois, 1409 W. Green St., Urbana IL 61801 USA

E-mail: diamond@math.uiuc.edu; heini@math.uiuc.edu

Received: 17 November 2006; revised: 3 February 2007 